Andrew X. Zhu, Massachusetts General Hospital Cancer Center, Harvard Medical School, Boston, MA; Olivier Rosmorduc, Service d'Hépatologie, Hôpital Saint-Antoine, Paris; Marie-Aude Leberre, Bayer HealthCare Pharmaceuticals, Loos, France; T.R. Jeffry Evans, Beatson West of Scotland Cancer Centre, Institute of Cancer Sciences, University of Glasgow, Glasgow; Paul J. Ross, King's College Hospital, London, United Kingdom; Armando Santoro, Humanitas Cancer Center, Milan, Italy; Flair Jose Carrilho, University of São Paulo School of Medicine, São Paulo, Brazil; Jordi Bruix and Josep M. Llovet, Barcelona Clínic Liver Cancer Group, Institut d'Investigacions Biomèdiques, August Pi i Sunyer, Hospital Clínic Barcelona, Centro de Investigación Biomédica en Red de Enfermedades Hepáticas y Digestivas, Barcelona; Josep M. Llovet, Institució Catalana de Recerca I Estudis Avançats, Catalonia, Spain; Shukui Qin, People's Liberation Army Cancer Center of Nanjing Bayi Hospital, Jiangsu, China; Paul J. Thuluvath, Institute for Digestive Health and Liver Diseases, Mercy Medical Center, Baltimore, MD; Josep M. Llovet, Mount Sinai Liver Cancer Program, Mount Sinai School of Medicine, New York, NY; Markus Jensen, Bayer Vital GmbH Leverkusen, Germany; Gerold Meinhardt, Bayer HealthCare Pharmaceuticals, Montville, NJ; and Yoon-Koo Kans, University of Ulsan College of Medicine, Asan Medical Center, Seoul, Republic of Korea.

Published online ahead of print at www.jco.org on December 29, 2014

A.X.Z. and Y.-K.K. contributed equally to this study

Authors' disclosures of potential conflicts of interest and author contributions are found at the end of this article.

Clinical trial information: NCT0901901.

Corresponding author: Andrew X. Zhu, MD, Massachusetts General Hospital Cancer Center, Harvard Medical School, 55 Fruit St, LH/POB 232, Boston, MA 02114; e-mail: azhu@partners.org.

(C) 2014 by American Society of Clinical Oncology

0732-183X/15/3306w-559w/\$20.00 DOI: $10.1200 / J C O .2013 .53 .7746$

\title{
SEARCH: A Phase III, Randomized, Double-Blind, Placebo-Controlled Trial of Sorafenib Plus Erlotinib in Patients With Advanced Hepatocellular Carcinoma
}

Andrew X. Zhu, Olivier Rosmorduc, T.R. Jeffry Evans, Paul J. Ross, Armando Santoro, Flair Jose Carrilho, Jordi Bruix, Shukui Qin, Paul J. Thuluvath, Josep M. Llovet, Marie-Aude Leberre, Markus Jensen, Gerold Meinhardt, and Yoon-Koo Kang

\section{$\begin{array}{llllllll}\text { A } & \text { B } & \text { S } & \text { T } & \text { R } & \text { A } & \text { C } & \text { T }\end{array}$}

\section{Purpose}

To compare the clinical outcomes of sorafenib plus either erlotinib or placebo in patients with advanced hepatocellular carcinoma (HCC) in a multicenter, multinational, randomized, phase III trial.

\section{Patients and Methods}

Patients with advanced HCC and underlying Child-Pugh class A cirrhosis, who were naive to systemic treatment $(N=720)$, were randomly assigned to sorafenib plus either erlotinib $(n=362)$ or placebo ( $n=358$ ). The primary end point was overall survival (OS).

\section{Results}

Median OS was similar in the sorafenib plus erlotinib and sorafenib plus placebo groups (9.5 v8.5 months, respectively; hazard ratio $[\mathrm{HR}], 0.929 ; P=.408)$, as was median time to progression (3.2 $\vee 4.0$ months, respectively; HR, 1.135; $P=.18$ ). In the sorafenib/erlotinib arm versus the sorafenib/placebo arm, the overall response rate trended higher $(6.6 \% \vee 3.9 \%$, respectively; $P=.102)$, whereas the disease control rate was significantly lower $(43.9 \% \vee 52.5 \%$, respectively; $P=.021)$. The median durations of treatment with sorafenib were 86 days in the sorafenib/erlotinib arm and 123 days in the sorafenib/placebo arm. In the sorafenib/erlotinib and sorafenib/placebo arms, the rates of treatment-emergent serious AEs $(58.0 \% \mathrm{~V}$ $54.6 \%$, respectively) and drug-related serious AEs $(21.0 \% \vee 22.8 \%$, respectively) were similar. AEs matched the known safety profiles of both agents, but rates of rash/desquamation, anorexia, and diarrhea were higher in the sorafenib/erlotinib arm, whereas rates of alopecia and hand-foot skin reaction were higher in the sorafenib/placebo arm. Withdrawal rates for AEs during cycles 1 to 3 were higher in the sorafenib/erlotinib arm.

\section{Conclusion}

Adding erlotinib to sorafenib did not improve survival in patients with advanced HCC.

\section{J Clin Oncol 33:559-566. (C) 2014 by American Society of Clinical Oncology}

\section{INTRODUCTION}

Hepatocellular carcinoma (HCC) is the third most frequent cause of cancer deaths worldwide. ${ }^{1}$ Most patients are diagnosed at advanced stages, when curative treatments, including resection, liver transplantation, and ablation, are no longer available. ${ }^{2,3}$ At present, sorafenib, a multikinase inhibitor, is the only systemic agent approved to treat advanced, unresectable $\mathrm{HCC}^{3-5}$ based on two phase III trials showing that sorafenib significantly improved survival in these patients. ${ }^{6,7}$

Other targeted agents, alone or combined with sorafenib, have been tested in patients with HCC.,9 The epidermal growth factor receptor (EGFR) pathway has been implicated in the pathogenesis of
HCC. ${ }^{10,11}$ EGFR activation may interfere with HCC response to sorafenib, ${ }^{11-13}$ suggesting that EGFR inhibition may enhance tumor response. Erlotinib is an orally active inhibitor of EGFR tyrosine kinase ${ }^{14,15}$ approved to treat patients with advanced nonsmall-cell lung and pancreatic cancers. ${ }^{16}$ Moreover, in two single-arm, phase II trials, erlotinib showed modest antitumor activity but promising overall survival (OS) benefit in patients with unresectable HCC. ${ }^{17,18}$

Because sorafenib and erlotinib target different pathways, treatment with both may have synergistic or additive inhibitory effects on tumor growth. In a phase I trial, the combination showed promising antitumor activity in patients with solid tumors, including HCC. ${ }^{19}$ To determine whether the addition 
of erlotinib improves the efficacy of sorafenib in patients with advanced HCC, a phase III trial compared the efficacy and safety of first-line sorafenib/erlotinib with sorafenib/placebo in these patients.

\section{PATIENTS AND METHODS}

\section{Patient Selection}

The SEARCH (Sorafenib and Erlotinib, a Randomized Trial Protocol for the Treatment of Patients With Hepatocellular Carcinoma) study was a randomized, double-blind, placebo-controlled, phase III trial conducted at 128 centers in 26 countries in Europe, North and South America, and the Asia-Pacific region, enrolling patients age $\geq 18$ years with histologically or radiologically confirmed advanced/metastatic HCC not amenable to local therapies. All patients had one or more measurable lesions according to RECIST, Child-Pugh class A (determined during screening), life expectancy $\geq 12$ weeks, and Eastern Cooperative Oncology Group (ECOG) performance status of 0 or 1 . Patients who previously received local therapy (eg, surgery, transcatheter arterial chemoembolization, or radiofrequency ablation) were eligible if therapy was completed $\geq$ 4 weeks before baseline scan and the previously treated lesions could not be selected as target lesions. All patients had adequate hematologic, hepatic, and kidney function, including a platelet count $\geq 60 \times 10^{9} / \mathrm{L}$, hemoglobin $\geq 8.5 \mathrm{~g} / \mathrm{dL}$, total bilirubin $\leq 2.8 \mathrm{mg} / \mathrm{dL}$, ALT and AST $\leq 5 \times$ the upper limit of normal, serum creatinine $\leq 1.5 \times$ the upper limit of normal, and prothrombin time-international normalized ratio $\leq 2.3$ or prothrombin time $\leq 6$ seconds above control.

Exclusion criteria included the following: prior systemic treatment for HCC; concurrent cancer (except for squamous cell carcinoma in situ, cervical cancer, superficial bladder cancer or cancer cured $>3$ years before trial entry); renal failure requiring hemodialysis or peritoneal dialysis; history of cardiovascular or peripheral vascular disease; active, serious clinical infection (except for hepatitis $\mathrm{B}$ or $\mathrm{C}$ virus); interstitial lung disease; previous treatment with yttrium-90 spheres; treatment with biologic response modifiers (eg, growth factors) within 3 weeks of trial entry; major surgery within 4 weeks; radiotherapy within 3 weeks; proton pump inhibitors within 2 weeks; and any condition that would interfere with study participation or data interpretation. Medications prohibited during the trial included proton pump inhibitors, strong inhibitors of CYP3A4, and biologic response modifiers (except to manage acute toxicities).

The study protocol was approved by the institutional review board of each center. All patients provided written informed consent before studyrelated procedures.

\section{Random Assignment}

Patients were randomly assigned 1:1 to treatment with sorafenib/ erlotinib or sorafenib/placebo, with random assignment stratified by ECOG performance status $(0 v 1)$, macrovascular invasion (MVI) and/or extrahepatic spread (EHS; yes $v$ no), smoking status (current $v$ former $v$ never), and geographic region (North/South America $v$ Europe/South Africa $v$ AsiaPacific). A computer-generated random assignment list was provided in a blinded fashion to the Interactive Voice Response System/Interactive Web Response System vendor, with random assignment based on a parallel preallocated balanced (same weighting per block) design with a block size of four. The Interactive Voice Response System/Interactive Web Response System assigned each eligible patient a random assignment number based on treatment allocation and stratification factors and provided the pack number of sorafenib and erlotinib or placebo to dispense.

\section{Treatment}

Patients were treated with sorafenib $400 \mathrm{mg}$ twice a day plus either erlotinib or placebo $150 \mathrm{mg}$ once a day. Patients with treatment-related toxicities were allowed stepwise dose delays or reductions, to $400 \mathrm{mg}$ once every day and $400 \mathrm{mg}$ once every other day for sorafenib and to $100 \mathrm{mg}$ once every day and $50 \mathrm{mg}$ once every day for erlotinib or placebo. Patients with grade 1 or 2 toxicities were allowed supportive therapy, if available, before consideration of dose reduction. In patients who experienced grade 3 hematologic toxicities, study drugs were reduced alternately for every episode of this adverse event (AE), in a step-by-step fashion, starting with erlotinib or placebo, until resolution to grade $\leq 2$. The same rule was applied in patients with grade 4 hematologic or grade 3 nonhematologic toxicities, except that treatment with both drugs was interrupted until resolution to grade $\leq 2$ before recommencing at reduced doses. Patients with any grade 4 nonhematologic toxicity had treatment discontinued.

Erlotinib was interrupted in patients with any grade of suspected or proven keratitis or suspected interstitial lung disease; if the latter was confirmed, both drugs were permanently discontinued. In patients with grade 3 hypertension, both drugs were interrupted until resolution to grade $\leq 2$, followed by reinitiation at a reduced dose of sorafenib and unchanged dose of erlotinib. For subsequent episodes of grade 3 hypertension, study drugs were reduced alternately step by step, starting with erlotinib for the second episode. All patients were monitored for hypertension and treated with antihypertensive drugs.

For patients with grade 3 hand-foot skin reaction (HFSR) and other nonhematologic toxicities, both drugs were interrupted until resolution to grade $\leq 2$ before reinitiation at reduced doses. Study drugs were reduced step by step starting with erlotinib or placebo for the first episode and sorafenib for a second episode. If two or more toxicities occurred at the same time, the drug reduced was the one deemed by the investigator most likely to have caused the toxicity. Specified dose interruptions were for $\leq 30$ days; patients without sufficient resolution after 30 days were withdrawn.

Treatment compliance was monitored by each patient keeping a Drug Accountability Form, which included records of the receipt, distribution, and return of all study medications. The numbers of tablets dispensed to and returned by each patient were recorded.

\section{Assessments}

The primary end point was OS, defined as time from random assignment to death from any cause; patients remaining alive were censored at last contact date. A secondary end point was time to progression (TTP), defined as time from random assignment to radiologic disease progression, with patients censored at last date of tumor evaluation; patients who changed therapy before radiologic progression were censored at the last date of tumor evaluation while on study medication. Other secondary end points included disease control rate (DCR), defined as the percentage of patients with best response of complete response, partial response, or stable disease, according to RECIST criteria, for $\geq 28$ days; overall response rate (ORR), defined as the percentage of patients with best response of complete or partial response for $\geq 28$ days; and safety. Safety was assessed every 3 weeks, and radiologic tumor progression was assessed every 6 weeks. The intensity and severity of AEs were graded according to National Cancer Institute Common Terminology Criteria for Adverse Events (version 3.0). Serious AEs were defined as untoward medical occurrences that resulted in death, were life threatening, required inpatient hospitalization, prolonged hospitalization, resulted in persistent or significant disability or incapacity, or were important medical events. Radiologic disease progression was not centrally reviewed.

\section{Statistical Methods and Analyses}

Efficacy was analyzed in the intent-to-treat population, defined as all randomly assigned patients, and safety was analyzed in all patients who received $\geq$ one dose of study medication. Sample size calculations were based on OS, with a clinically meaningful improvement defined as an OS rate $33 \%$ higher in the sorafenib/erlotinib group than in the sorafenib/placebo group. Assuming a median OS for sorafenib/placebo of 10.7 months, the median OS for sorafenib/erlotinib would have to be $\geq 14.3$ months, and the hazard ratio (HR) for sorafenib/erlotinib versus sorafenib/placebo would have to be 0.752 . With a two-sided $\alpha=.05$, a power of $90 \%$, a 1:1 random assignment ratio, and one formal interim analysis of OS, using an O'Brien-Fleming-type error spending function, the study required approximately 521 events (deaths), and approximately 700 patients were planned to be randomly assigned. 


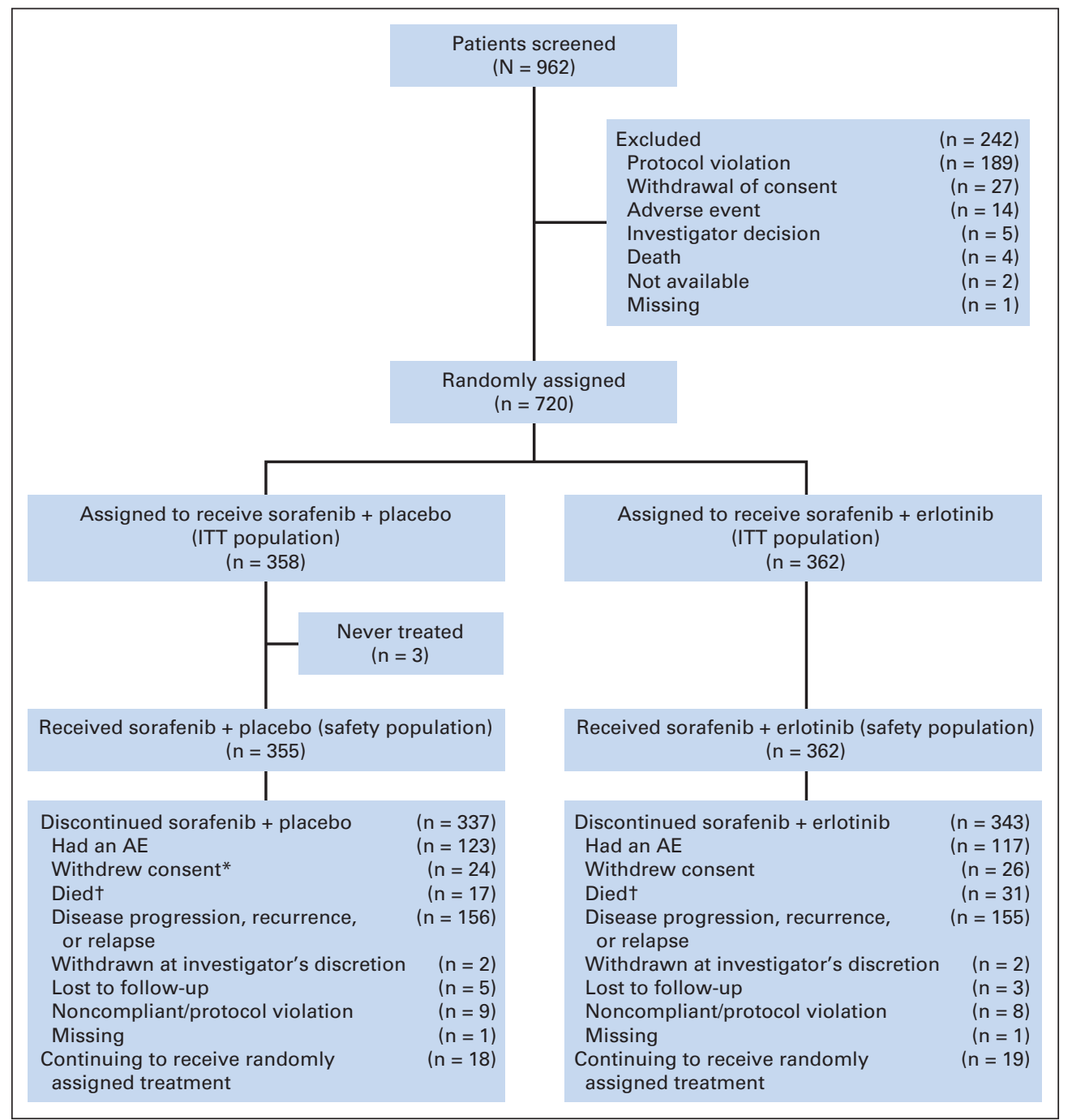

Fig 1. Patient disposition. $\left(^{*}\right)$ Not including one patient who withdrew consent without receiving study drug. (†) Not equivalent to the number of patients who died on study. See Table 4. AE, adverse event; ITT, intent to treat.

OS and TTP in the two treatment groups were compared using twosided log-rank tests with an overall $\alpha=.05$ stratified by ECOG performance status ( $0 v 1$ ), MVI and/or EHS (presence $v$ absence), and geographic region (North/South America $v$ Europe/South Africa $v$ Asia-Pacific). Kaplan-Meier estimates and survival curves were generated for each treatment group. In addition, HRs and corresponding 95\% CIs were obtained by a Cox model that included only treatment, with stratification by the previously described factors. An exploratory analysis of progression-free survival was performed similarly.

Tumor response was evaluated using RECIST version 1.0 criteria, with DCR and ORR compared using the Cochran-Mantel-Haenszel test, stratified as for OS. OS in subgroups was analyzed similarly to the overall intent-to-treat population. $P$ values for subgroup analyses are provided for descriptive purposes only. All $P$ values reported are two sided.

\section{RESULTS}

\section{Patient Characteristics and Disposition}

From May 2009 to January 2011, 962 patients were screened; 720 patients met the eligibility criteria and were randomly assigned, 362 to the sorafenib/erlotinib group and 358 to the sorafenib/placebo group
(Fig 1); and 362 and 355 patients, respectively, received at least one dose of study medication. Overall, the two groups were well matched in demographic and clinical characteristics (Table 1).

\section{Efficacy End Points}

Median OS was 9.5 months for the sorafenib/erlotinib group and 8.5 months for the sorafenib/placebo group (HR, 0.929; 95\% CI, 0.781 to $1.106 ; P=.408$; Fig $2 \mathrm{~A}$ ), and median TTP was 3.2 and 4.0 months, respectively (HR, $1.135 ; 95 \% \mathrm{CI}, 0.944$ to $1.366 ; P=.18$; Fig $2 \mathrm{~B})$. A post hoc exploratory analysis of progression-free survival showed results comparable to TTP (HR, 1.111; 95\% CI, 0.941 to 1.311 ).

ORR trended higher in the sorafenib/erlotinib arm than in the sorafenib/placebo arm (6.6\% v3.9\%, respectively; $P=.102$; Table 2$)$. In contrast, DCR was significantly higher in the sorafenib/ placebo arm than the sorafenib/erlotinib arm (52.5\% v 43.9\%, respectively; $P=.021$ ).

Subgroup analyses of OS by geographic region (North/South America, Europe, or Asia-Pacific), disease etiology (hepatitis C, hepatitis B, or alcohol), and MVI or EHS (yes or no) showed no significant 


\begin{tabular}{|c|c|c|c|c|}
\hline \multirow[b]{2}{*}{ Characteristic } & \multicolumn{2}{|c|}{$\begin{array}{c}\text { Sorafenib + } \\
\text { Placebo } \\
(n=358)\end{array}$} & \multicolumn{2}{|c|}{$\begin{array}{c}\text { Sorafenib + } \\
\text { Erlotinib } \\
(n=362)\end{array}$} \\
\hline & $\begin{array}{l}\text { No. of } \\
\text { Patients }\end{array}$ & $\%$ & $\begin{array}{l}\text { No. of } \\
\text { Patients }\end{array}$ & $\%$ \\
\hline Median age, years & \multicolumn{2}{|c|}{60.0} & \multicolumn{2}{|c|}{60.5} \\
\hline Male & 286 & 79.9 & 295 & 81.5 \\
\hline Liver cirrhosis & 251 & 70.1 & 240 & 66.3 \\
\hline Ascites & 36 & 10.1 & 40 & 11.0 \\
\hline Macroscopic vascular invasion & 153 & 42.7 & 138 & 38.1 \\
\hline Extrahepatic spread & 219 & 61.2 & 205 & 56.6 \\
\hline \multicolumn{5}{|l|}{ Etiology } \\
\hline Hepatitis B & 133 & 37.2 & 122 & 33.7 \\
\hline Hepatitis C & 84 & 23.5 & 107 & 29.6 \\
\hline \multicolumn{5}{|l|}{ BCLC stage } \\
\hline B & 48 & 13.4 & 60 & 16.6 \\
\hline C & 310 & 86.6 & 302 & 83.4 \\
\hline \multicolumn{5}{|l|}{ Child-Pugh score } \\
\hline 5 & 252 & 70.4 & 246 & 68.0 \\
\hline 6 & 93 & 26.0 & 110 & 30.4 \\
\hline \multicolumn{5}{|l|}{ ECOG PS } \\
\hline 0 & 216 & 60.3 & 222 & 61.3 \\
\hline 1 & 142 & 39.7 & 140 & 38.7 \\
\hline \multicolumn{5}{|l|}{ Geographic region } \\
\hline North or South America & 85 & 23.7 & 88 & 24.3 \\
\hline Europe & 183 & 51.1 & 186 & 51.4 \\
\hline Asia-Pacific & 90 & 25.1 & 88 & 24.3 \\
\hline \multicolumn{5}{|l|}{ Smoking status } \\
\hline Never & 107 & 29.9 & 112 & 30.9 \\
\hline Former & 128 & 35.8 & 132 & 36.5 \\
\hline Current & 123 & 34.4 & 118 & 32.6 \\
\hline
\end{tabular}

differences between the sorafenib/erlotinib and sorafenib/placebo groups (Fig 3). TTP in the stratified subgroups also did not differ significantly (data not shown). Analysis by smoking status showed that median OS was approximately 3 months longer for former

\begin{tabular}{|c|c|c|c|c|c|}
\hline \multirow[b]{2}{*}{ Best Response } & \multicolumn{2}{|c|}{$\begin{array}{c}\text { Sorafenib + } \\
\text { Placebo } \\
(n=358)\end{array}$} & \multicolumn{2}{|c|}{$\begin{array}{c}\text { Sorafenib + } \\
\text { Erlotinib } \\
(n=362)\end{array}$} & \multirow[b]{2}{*}{$P^{*}$} \\
\hline & $\begin{array}{c}\text { No. of } \\
\text { Patients }\end{array}$ & $\%$ & $\begin{array}{l}\text { No. of } \\
\text { Patients }\end{array}$ & $\%$ & \\
\hline$\overline{O R R}(\mathrm{CR}+\mathrm{PR}) \dagger$ & 14 & 3.9 & 24 & 6.6 & .102 \\
\hline $\mathrm{CR}$ & 1 & 0.3 & 2 & 0.6 & \\
\hline PR & 13 & 3.6 & 22 & 6.1 & \\
\hline SD & 174 & 48.6 & 135 & 37.3 & \\
\hline PD & 119 & 33.2 & 138 & 38.1 & \\
\hline $\mathrm{DCR}(\mathrm{CR}+\mathrm{PR}+\mathrm{SD})$ & 188 & 52.5 & 159 & 43.9 & .021 \\
\hline Not assessable & 51 & 14.2 & 65 & 18.0 & Not assessable \\
\hline \multicolumn{6}{|c|}{$\begin{array}{l}\text { Abbreviations: CR, complete response; DCR, disease control rate; ORR, } \\
\text { overall response rate; PD, progressive disease; PR, partial response; } S D \text {, } \\
\text { stable disease. } \\
\text { "Two-sided Cochran-Mantel-Haenszel test. } \\
\text { tIntent-to-treat population. Fifty-one patients in the sorafenib plus placebo } \\
\text { group and } 65 \text { patients in the sorafenib plus erlotinib were not assessable } \\
\text { for response. }\end{array}$} \\
\hline
\end{tabular}

smokers $(\mathrm{n}=260)$ than for current smokers $(\mathrm{n}=242)$ and neversmokers $(n=219)$, independent of treatment arm.

\section{Treatment Duration and Dosing}

The median daily doses per cycle of sorafenib in the sorafenib/ erlotinib and sorafenib/placebo groups were 773 and $768 \mathrm{mg}$, respectively, with mean daily doses of $678.9 \pm 161.3$ and $673.1 \pm$ $156.2 \mathrm{mg}$, respectively. The median and mean daily doses per cycle for erlotinib in the sorafenib/erlotinib group were 143 and $124.4 \pm$ $32.4 \mathrm{mg}$, respectively, whereas the median and mean daily doses of placebo in the sorafenib/placebo group were 142 and $128.2 \pm 43.0$ $\mathrm{mg}$, respectively.

The median durations of sorafenib treatment in the sorafenib/ erlotinib and sorafenib/placebo groups were 86 and 123 days, respectively. The median and mean durations of treatment with erlotinib for patients in the sorafenib/erlotinib group were 86 and 157 days, respectively.

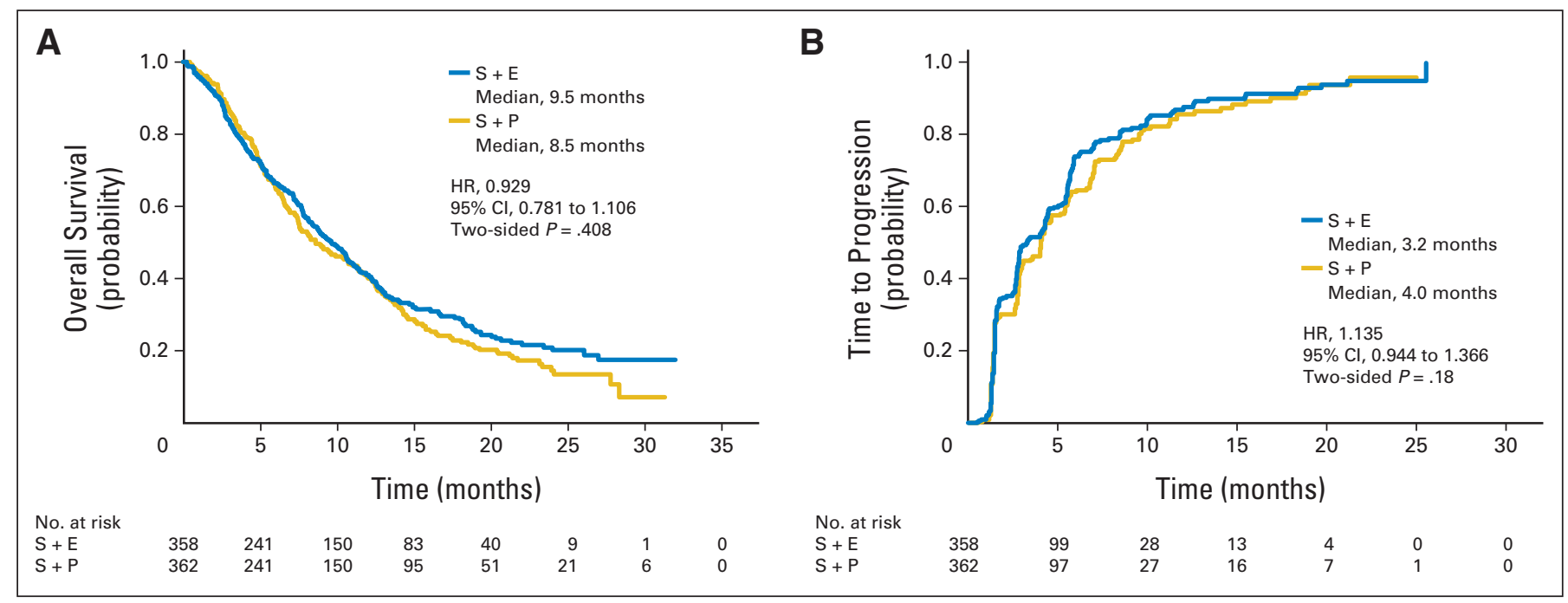

Fig 2. Kaplan-Meier curves of (A) overall survival (OS) and (B) time to progression (TTP) in the sorafenib plus erlotinib (S + E) and sorafenib plus placebo (S + P) groups. There were 523 events in the OS analysis (255 in the S + E group and 268 in the S + P group) and 469 events in the TTP analysis (240 in the S + E group and 229 in the S + P group). HR, hazard ratio. 


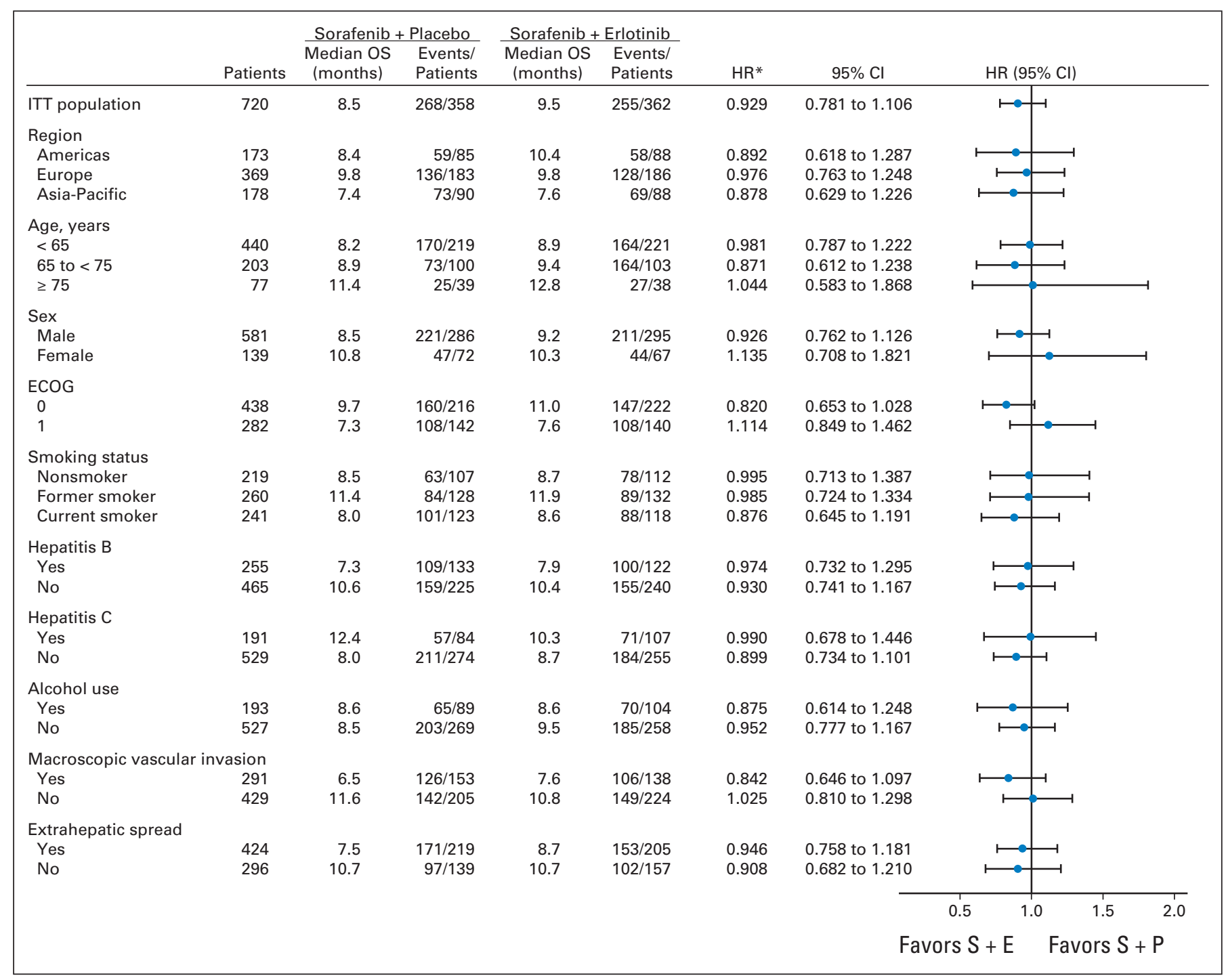

Fig 3. Forest plot showing subgroup analyses of overall survival. $\left(^{*}\right)$ Calculated for sorafenib + erlotinib/sorafenib + placebo. ECOG, Eastern Cooperative Oncology Group; HR, hazard ratio; ITT, intent to treat; OS, overall survival; $S+E$, sorafenib plus erlotinib; $S+P$, sorafenib plus placebo.

Sorafenib doses in the sorafenib/erlotinib and sorafenib/placebo groups were interrupted in 300 patients $(82.9 \%)$ and 285 patients (79.6\%), respectively, and were reduced in 121 patients $(33.4 \%)$ and 136 patients $(38.0 \%)$, respectively. Erlotinib and placebo doses were interrupted in 258 patients ( $85.4 \%$ ) and 252 patients $(87.5 \%)$, respectively, and were reduced in 217 patients (59.9\%) and 211 patients (58.9\%), respectively. Dosing and dose modifications by geographic region are shown in Table 3.

\section{Safety}

In the sorafenib/erlotinib and sorafenib/placebo groups, global rates of treatment-emergent AEs (100\% v 99.2\%, respectively), drugrelated AEs ( $95.0 \% \vee 95.2 \%$, respectively), treatment-emergent serious AEs (58.0\% $v 54.6 \%$, respectively), and drug-related serious AEs (21.0\% $v 22.8 \%$, respectively) were similar.

Table 4 lists the incidence of treatment-emergent AEs affecting $\geq$ $15 \%$ of patients in either group and the incidence of corresponding grade 3 , 4, or 5 AEs. Although the overall AE profiles of the two groups were similar, there were noticeable differences. AEs that were more than $5 \%$ more frequent in the sorafenib/erlotinib group versus sorafenib/placebo group included rash/desquamation $(51.9 \% v$ $40.0 \%$, respectively), anorexia (42.5\% $v 37.2 \%$, respectively), diarrhea (76.2\% $v$ 59.4\%, respectively), and nose bleeding (17.4\% $v 7.0 \%$, respectively), whereas AEs that were more than $5 \%$ more frequent in the sorafenib/placebo group versus the sorafenib/erlotinib group included alopecia (23.7\% v 12.7\%, respectively), HFSR (47.6\% v38.1\%, respectively), constipation $(20.0 \% \vee 12.7 \%$, respectively), cough (16.9\% $v 11.0 \%$, respectively), and nausea (31.0\% $v 24.3 \%$, respectively). The incidence of most treatment-emergent serious AEs was less than $5 \%$ for the entire population, with exceptions possibly reflecting disease state rather than drug-relatedness, including hepatobiliary/pancreas $(10.7 \%)$, constitutional symptoms $(8.6 \%)$, infection $(7.9 \%)$, death (7.0\%), pain (7.0\%), and metabolic/laboratory (5.4\%). Individual serious $\mathrm{AE}$ categories with differences across treatment arms of more than $2 \%$ (sorafenib/erlotinib $v$ sorafenib/placebo) were death $(8.0 \% v 5.9 \%$, respectively), infection ( $6.6 \% \vee 9.3 \%$, respectively), metabolic/laboratory 


\begin{tabular}{|c|c|c|c|c|c|c|}
\hline \multirow[b]{2}{*}{ Parameter } & \multicolumn{2}{|c|}{$\begin{array}{l}\text { Americas } \\
(n=173)\end{array}$} & \multicolumn{2}{|c|}{$\begin{array}{c}\text { Europe } \\
(n=369)\end{array}$} & \multicolumn{2}{|c|}{$\begin{array}{l}\text { Asia-Pacific } \\
(n=178)\end{array}$} \\
\hline & $S+P$ & $S+E$ & $S+P$ & $S+E$ & $S+P$ & $S+E$ \\
\hline \multicolumn{7}{|l|}{ Sorafenib } \\
\hline Median daily dose, mg & 751 & 764 & 758 & 799 & 776 & 749 \\
\hline Mean daily dose, mg & 667 & 667 & 670 & 694 & 686 & 660 \\
\hline Dose interruption, \% & 82.4 & 85.2 & 80.9 & 84.9 & 74.4 & 76.1 \\
\hline Dose reduction, \% & 48.2 & 46.6 & 32.8 & 23.1 & 38.9 & 42.0 \\
\hline \multicolumn{7}{|l|}{ Erlotinib } \\
\hline Median daily dose, mg & & 139 & & 149 & & 132 \\
\hline Mean daily dose, mg & & 123 & & 126 & & 121 \\
\hline Dose interruption, \% & & 84.1 & & 86.6 & & 76.1 \\
\hline Dose reduction, \% & & 48.9 & & 34.9 & & 42.0 \\
\hline \multicolumn{7}{|l|}{ Placebo } \\
\hline Median daily dose, mg & 138 & & 142 & & 145 & \\
\hline Mean daily dose, mg & 127 & & 128 & & 130 & \\
\hline Dose interruption, \% & 84.7 & & 80.9 & & 75.6 & \\
\hline Dose reduction, \% & 42.4 & & 40.4 & & 41.1 & \\
\hline
\end{tabular}

Abbreviations: E, erlotinib; P, placebo; $S$, sorafenib.

(6.6\% $v 4.2 \%$, respectively), neurology ( $3.0 \% v 5.6 \%$, respectively), and GI $(15.2 \% v 10.1 \%$, respectively), with diarrhea accounting for the biggest difference ( $4.1 \% v 0.8 \%$, respectively).

Death rates in the sorafenib/erlotinib and sorafenib/placebo groups were similar during the first 30 days of treatment $(4.1 \% v 2.5 \%$, respectively) and up to 30 days after the last dose of study medication $(21.0 \% \vee 18.3 \%$, respectively), with the single highest number of deaths being a result of liver dysfunction ( $20 v 18$ deaths, respectively). Drug-related deaths attributed to sorafenib in the sorafenib/erlotinib and sorafenib/placebo groups (nine $v 11$ deaths, respectively) and those attributed to erlotinib or placebo (10v11 deaths, respectively) were also similar, with the highest number of deaths caused by liver dysfunction (two $v$ four deaths in the sorafenib/erlotinib $v$ sorafenib/ placebo group, respectively). Deaths without progression were also comparable in the sorafenib/erlotinib and sorafenib/placebo groups ( $53 v 59$ deaths, respectively).

\section{Withdrawal From Treatment}

The incidence of treatment-emergent AEs leading to withdrawal of any drug was similar in the sorafenib/erlotinib $(\mathrm{n}=163 ; 45.0 \%)$ and sorafenib/placebo $(n=162 ; 45.6 \%)$ groups. Patients in the former group tended to withdraw from sorafenib earlier as a results of AEs, with 99 patients $(27.3 \%)$ receiving sorafenib/erlotinib and 83 patients (23.4\%) receiving sorafenib/placebo failing to complete three cycles of sorafenib as a result of AEs, and 103 patients (28.5\%) and 84 patients (23.7\%), respectively, failing to complete three cycles of erlotinib or placebo as a result of AEs. Causes of withdrawal with an incidence of $\geq 2 \%$ in the sorafenib/erlotinib and sorafenib/placebo groups included fatigue $(4.1 \% v 0.8 \%$, respectively), diarrhea ( $3.3 \% \vee 0.8 \%$, respectively), and dermatologic $(5.0 \% v 2.3 \%$, respectively).

\begin{tabular}{|c|c|c|c|c|c|c|c|c|c|c|c|c|c|c|c|c|}
\hline \multirow[b]{3}{*}{$A E$} & \multicolumn{8}{|c|}{ Sorafenib Plus Placebo $(n=355)$} & \multicolumn{8}{|c|}{ Sorafenib Plus Erlotinib $(n=362)$} \\
\hline & \multicolumn{2}{|c|}{ All Grades } & \multicolumn{2}{|c|}{ Grade 3} & \multicolumn{2}{|c|}{ Grade 4} & \multicolumn{2}{|c|}{ Grade 5} & \multicolumn{2}{|c|}{ All Grades } & \multicolumn{2}{|c|}{ Grade 3} & \multicolumn{2}{|c|}{ Grade 4} & \multicolumn{2}{|c|}{ Grade 5} \\
\hline & $\begin{array}{l}\text { No. of } \\
\text { Patients }\end{array}$ & $\%$ & $\begin{array}{c}\text { No. of } \\
\text { Patients }\end{array}$ & $\%$ & $\begin{array}{c}\text { No. of } \\
\text { Patients }\end{array}$ & $\%$ & $\begin{array}{c}\text { No. of } \\
\text { Patients }\end{array}$ & $\%$ & $\begin{array}{l}\text { No. of } \\
\text { Patients }\end{array}$ & $\%$ & $\begin{array}{c}\text { No. of } \\
\text { Patients }\end{array}$ & $\%$ & $\begin{array}{l}\text { No. of } \\
\text { Patients }\end{array}$ & $\%$ & $\begin{array}{c}\text { No. of } \\
\text { Patients }\end{array}$ & $\%$ \\
\hline Any event & 352 & 99.2 & 180 & 50.7 & 46 & 13.0 & 72 & 20.3 & 362 & 100.0 & 179 & 49.4 & 56 & 15.5 & 80 & 22.1 \\
\hline Diarrhea & 211 & 59.4 & 42 & 11.8 & 0 & 0 & & & 276 & 76.2 & 89 & 18.5 & 3 & 0.8 & & \\
\hline Fatigue & 191 & 53.8 & 57 & 16.1 & 5 & 1.4 & & & 186 & 51.4 & 62 & 17.1 & 2 & 0.6 & & \\
\hline Hand-foot skin reaction & 169 & 47.6 & 62 & 17.5 & & & & & 138 & 38.1 & 37 & 10.2 & & & & \\
\hline Rash/desquamation & 142 & 40.0 & 16 & 4.5 & 0 & 0 & & & 188 & 51.9 & 31 & 8.6 & 2 & 0.6 & & \\
\hline Anorexia & 132 & 37.2 & 20 & 5.6 & & & & & 154 & 42.5 & 15 & 4.1 & & & & \\
\hline Pain, abdomen NOS & 115 & 32.4 & 24 & 6.8 & 1 & 0.3 & & & 116 & 32.0 & 21 & 5.8 & 1 & 0.3 & & \\
\hline Nausea & 110 & 31.0 & 10 & 2.8 & & & & & 88 & 24.3 & 7 & 1.9 & & & & \\
\hline Weight loss & 109 & 30.7 & 13 & 3.7 & & & & & 126 & 34.8 & 15 & 4.1 & & & & \\
\hline Ascites & 89 & 25.1 & 31 & 8.7 & 0 & 0 & & & 81 & 22.4 & 33 & 9.1 & 2 & 0.6 & & \\
\hline Hypertension & 87 & 24.5 & 31 & 8.7 & & & & & 71 & 19.6 & 17 & 4.7 & & & & \\
\hline Alopecia & 84 & 23.7 & & & & & & & 46 & 12.7 & & & & & & \\
\hline Vomiting & 81 & 22.8 & 7 & 2.0 & 0 & 0 & & & 79 & 21.8 & 7 & 1.9 & 1 & 0.3 & 0 & \\
\hline Edema, limb & 73 & 20.6 & 5 & 1.4 & & & & & 81 & 22.4 & 4 & 1.1 & & & & \\
\hline Constipation & 71 & 20.0 & 4 & 1.1 & & & & & 46 & 12.7 & 2 & 0.6 & & & & \\
\hline Fever & 70 & 19.7 & 1 & 0.3 & & & & & 77 & 21.3 & 2 & 0.6 & & & & \\
\hline Cough & 60 & 16.9 & & & & & & & 40 & 11.0 & & & & & & \\
\hline Mucositis, oral & 53 & 14.9 & 3 & 0.8 & & & & & 72 & 19.9 & 7 & 1.9 & & & & \\
\hline Hemorrhage, nose & 25 & 7.0 & 0 & 0 & & & & & 63 & 17.4 & 1 & 0.3 & & & & \\
\hline \multicolumn{17}{|l|}{ Metabolic/laboratory } \\
\hline Bilirubin (hyperbilirubinemia) & 76 & 21.4 & 32 & 9.0 & 9 & 2.5 & & & 68 & 18.8 & 29 & 8.0 & 14 & 3.9 & & \\
\hline AST & 75 & 21.1 & 38 & 10.7 & 4 & 1.1 & & & 79 & 21.8 & 43 & 11.9 & 7 & 1.9 & & \\
\hline Hemoglobin & 50 & 14.1 & 15 & 4.2 & 6 & 1.7 & & & 68 & 18.8 & 23 & 6.4 & 6 & 1.7 & & \\
\hline
\end{tabular}




\section{DISCUSSION}

Despite sorafenib improving OS in patients with advanced HCC, most patients ultimately succumb. This trial, testing whether the combination of sorafenib and erlotinib improved outcomes in patients with unresectable HCC, did not meet its primary end point, an improvement in OS. Furthermore, this combination did not significantly prolong TTP.

Although phase II studies reported that erlotinib monotherapy had activity in patients with advanced HCC, ${ }^{17,18}$ combining erlotinib with sorafenib did not enhance efficacy compared with sorafenib alone. Although ORR trended higher in the sorafenib/erlotinib arm than in the sorafenib/placebo arm $(P=$ .102 ), suggesting that the combination may result in greater tumor shrinkage in some patients, the higher response rate did not translate into a prolongation of OS or TTP.

In contrast, DCR (which includes ORR and stable disease) was significantly lower in the sorafenib/erlotinib group than in the sorafenib/placebo group $(P=.021)$. Although the daily doses of sorafenib and erlotinib or placebo were similar in the two groups, treatment duration was shorter in the sorafenib/erlotinib group than in the sorafenib/placebo ( $2.8 \vee 4.0$ months, respectively). Although this finding suggested an association between treatment duration and DCR, causality could not be determined. Indeed, longer treatment duration with sorafenib has been associated with improved clinical outcomes in patients treated with sorafenib during ${ }^{20}$ or after ${ }^{21}$ transcatheter arterial chemoembolization.

More patients in the sorafenib/erlotinib group than in the sorafenib/placebo group discontinued treatment during cycles 1 to 3 . The rate of documented AEs leading to discontinuation was higher in the sorafenib/erlotinib group. From cycle 4 onward, there were no meaningful between-group differences in dropout rates.

Patients with non-small-cell lung cancer who actively smoke are less likely to respond to erlotinib and to experience erlotinib-related toxicity, as a result of its rapid metabolism and lower drug exposure. ${ }^{22,23}$ Interestingly, median OS was approximately 3 months longer in former smokers than in current and never-smokers, regardless of treatment arm. The mechanism underlying these differences is unknown.

Some tyrosine kinase inhibitor-associated AEs, including erlotinibassociated erythema ${ }^{24}$ and sorafenib-associated HFSR, ${ }^{25}$ may be more frequent during the first weeks of treatment, with severity and frequency decreasing thereafter. Sorafenib and erlotinib have overlapping skin toxicity profiles, so that additive skin toxicity may occur during the first two or three cycles of therapy, possibly resulting in withdrawals. This hypothesis is difficult to confirm, especially because an erlotinib monotherapy group was not available for comparison.

The overall incidence of AEs was similar in the two groups, although the incidence of AEs requiring withdrawal from treatment was slightly higher in the sorafenib/erlotinib group. The higher risk of AEs in the combination group may have been masked by the relatively longer treatment duration in the sorafenib/placebo group, explaining why some sorafenibassociated AEs occurred more frequently in this group.

Our study has several limitations. First, despite the scientific rationale for targeting EGFR in HCC and the promising results of phase II trials of erlotinib monotherapy, no phase II trials assessed the efficacy and safety of this combination in advanced HCC. Second, as reported in phase III trials of sunitinib, linifanib, and brivanib, ${ }^{26-29}$ statistical assumptions were not based on phase II combination data. Future phase III trials should select agents and regimens with proven tolerability and favorable safety profiles in HCC-specific phase I trials and documented efficacy based on well-designed, preferably randomized, phase II trials. ${ }^{30}$ The lack of synergistic or additive effect suggests that EGFR signaling may not be pivotal in advanced HCC.

In conclusion, adding erlotinib to sorafenib was associated with a shorter sorafenib treatment duration and did not improve survival in patients with advanced HCC. Sorafenib remains the standard of care for first-line treatment of advanced HCC.

\section{AUTHORS' DISCLOSURES OF POTENTIAL CONFLICTS OF INTEREST}

Although all authors completed the disclosure declaration, the following author(s) and/or an author's immediate family member(s) indicated a financial or other interest that is relevant to the subject matter under consideration in this article. Certain relationships marked with a " $U$ " are those for which no compensation was received; those relationships marked with a " $C$ " were compensated. For a detailed description of the disclosure categories, or for more information about ASCO's conflict of interest policy, please refer to the Author Disclosure Declaration and the Disclosures of Potential Conflicts of Interest section in Information for Contributors. Employment or Leadership Position: Marie-Aude Leberre, Bayer (C); Markus Jensen, Bayer (C); Gerold Meinhardt, Bayer (C) Consultant or Advisory Role: T.R. Jeffry Evans, Bayer (C); Paul J. Ross, Bayer (C), Bristol-Myers Squibb (C), Roche (C), Daiichi-Sankyo (C); Jordi Bruix, Daiichi Sankyo (C), ArQule (C), Bayer (C), Biocompatibles (C), Bristol-Myers Squibb (C), GlaxoSmithKline (C), Kowa (C), Eli Lilly (C), Novartis (C); Josep M. Llovet, Bayer (C), Bristol-Myers Squibb (C), Eli Lilly (C), Celsion (C), Biocompatibles (C), Novartis (C), Boehringer-Ingelheim (C), GlaxoSmithKline (C), Blueprint Medicines (C); Yoon-Koo Kang, Bayer (C), Pfizer (C), Novartis (C) Stock Ownership: Markus Jensen, Bayer; Gerold Meinhardt, Bayer Honoraria: Andrew X. Zhu, Exelixis, Celgene, sanofi-aventis, Eisai; Olivier Rosmorduc, Bayer, Roche; T.R. Jeffry Evans, Bayer; Paul J. Ross, Bayer; Jordi Bruix, Bayer, Biocompatibles, Bristol-Myers Squibb, Kowa; Paul J. Thuluvath, Onyx; Josep M. Llovet, Bayer Pharmaceuticals; Yoon-Koo Kang, Bayer, Pfizer, Novartis Research Funding: Andrew X. Zhu, Novartis, Bayer, Onyx; Flair Jose Carrilho, Bayer; Jordi Bruix, Daiichi Sankyo, ArQule, Bayer; Paul J. Thuluvath, Bayer; Josep M. Llovet, Boehringer-Ingelheim, Bayer Pharmaceuticals; Yoon-Koo Kang, Bayer, Novartis Expert Testimony: Paul J. Ross, Bayer (C) Patents, Royalties, and Licenses: None Other Remuneration: Paul J. Ross, Bayer, Roche

\section{AUTHOR CONTRIBUTIONS}

Conception and design: Andrew X. Zhu, Jordi Bruix, Josep M. Llovet, Markus Jensen, Gerold Meinhardt, Yoon-Koo Kang

Administrative support: Andrew X. Zhu

Provision of study materials or patients: Andrew X. Zhu, T.R. Jeffry Evans, Paul J. Ross, Yoon-Koo Kang

Collection and assembly of data: Andrew X. Zhu, Olivier Rosmorduc, T.R. Jeffry Evans, Paul J. Ross, Armando Santoro, Flair Jose Carrilho, Jordi Bruix, Shukui Qin, Paul J. Thuluvath, Josep M. Llovet, Yoon-Koo Kang

Data analysis and interpretation: Andrew X. Zhu, Olivier Rosmorduc, Armando Santoro, Marie-Aude Leberre, Markus Jensen, Gerold

Meinhardt, Yoon-Koo Kang

Manuscript writing: All authors

Final approval of manuscript: All authors 


\section{REFERENCES}

1. Jemal A, Bray F, Center MM, et al: Global cancer statistics. CA Cancer J Clin 61:69-90, 2011

2. Thomas MB, Jaffe D, Choti MM, et al: Hepatocellular carcinoma: Consensus recommendations of the National Cancer Institute Clinical Trials Planning Meeting. J Clin Oncol 28:3994-4005, 2010

3. National Comprehensive Cancer Network: NCCN Guidelines Version 2.2013, 2013. http://www.nccn.org/ professionals/physician_gls/f_guidelines.asp

4. Bayer HealthCare Pharmaceuticals: Nexavar (tablets, oral): Prescribing information. Wayne, $\mathrm{NJ}$, Bayer HealthCare Pharmaceuticals, 2012

5. Wilhelm SM, Carter $C$, Tang $L$, et al: BAY 43-9006 exhibits broad spectrum oral antitumor activity and targets the RAF/MEK/ERK pathway and receptor tyrosine kinases involved in tumor progression and angiogenesis. Cancer Res 64:7099-7109, 2004

6. Llovet JM, Ricci $S$, Mazzaferro $V$, et al Sorafenib in advanced hepatocellular carcinoma. N Engl J Med 359:378-390, 2008

7. Cheng AL, Kang YK, Chen Z, et al: Efficacy and safety of sorafenib in patients in the Asia-Pacific region with advanced hepatocellular carcinoma: A phase II randomised, double-blind, placebo-controlled trial. Lancet Oncol 10:25-34, 2009

8. Tazi el M, Essadi I, M'rabti $\mathrm{H}$, et al: Systemic treatment and targeted therapy in patients with advanced hepatocellular carcinoma. N Am J Med Sci 3:167-175, 2011

9. Zhu AX: Molecularly targeted therapy for advanced hepatocellular carcinoma in 2012: Current status and future perspectives. Semin Oncol 39: 493-502, 2012

10. Berasain $C$, Nicou A, Garcia-Irigoyen $O$, et al: Epidermal growth factor receptor signaling in hepatocellular carcinoma: Inflammatory activation and a new intracellular regulatory mechanism. Dig Dis 30:524-531, 2012
11. Bassullu N, Turkmen I, Dayangac M, et al: The predictive and prognostic significance of c-erb-B2, EGFR, PTEN, mTOR, PI3K, p27, and ERCC1 expression in hepatocellular carcinoma. Hepat Mon 12: e7492, 2012

12. Ezzoukhry Z, Louandre C, Trécherel E, et al: EGFR activation is a potential determinant of primary resistance of hepatocellular carcinoma cells to sorafenib. Int J Cancer 131:2961-2969, 2012

13. Blivet-Van Eggelpoël MJ, Chettouh H, Fartoux $\mathrm{L}$, et al: Epidermal growth factor receptor and HER-3 restrict cell response to sorafenib in hepatocellular carcinoma cells. J Hepatol 57:108-115, 2012

14. Iyer $R$, Bharthuar $A$ : A review of erlotinib-An oral, selective epidermal growth factor receptor tyrosine kinase inhibitor. Expert Opin Pharmacother 11:311-320, 2010

15. Steins $M$, Thomas $M$, Geissler M: Erlotinib. Recent Results Cancer Res 184:21-31, 2010

16. Astellas Pharma US, Genentech: Tarceva (erlotinib tablets, oral): Prescribing information. Farmingdale, NY, Astellas Pharma US, Genentech, 2012

17. Philip PA, Mahoney MR, Allmer $C$, et al: Phase II study of erlotinib (OSI-774) in patients with advanced hepatocellular cancer. J Clin Oncol 23:66576663, 2005

18. Thomas MB, Chadha R, Glover K, et al: Phase 2 study of erlotinib in patients with unresectable hepatocellular carcinoma. Cancer 110:1059-1067, 2007

19. Duran I, Hotté SJ, Hirte $H$, et al: Phase I targeted combination trial of sorafenib and erlotinib in patients with advanced solid tumors. Clin Cancer Res 13:4849-4857, 2007

20. Lencioni R, Llovet JM, Han G, et al: Sorafenib or placebo in combination with transarterial chemoembolization (TACE) with doxorubicin-eluting beads (DEBDOX) for intermediate-stage hepatocellular carcinoma (HCC): Phase II, randomized, double-blind SPACE trial. J Clin Oncol 30, 2012 (suppl; abstr LBA154)

21. Kudo M, Imanaka K, Chida N, et al: Phase III study of sorafenib after transarterial chemoembolisation in Japanese and Korean patients with unre- sectable hepatocellular carcinoma. Eur J Cancer 47:2117-2127, 2011

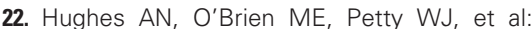
Overcoming CYP1A1/1A2 mediated induction of metabolism by escalating erlotinib dose in current smokers. J Clin Oncol 27:1220-1226, 2009

23. Waller LL, Miller AA, Petty WJ: Using erlotinib to treat patients with non-small cell lung cancer who continue to smoke. Lung Cancer 67:12-16, 2010

24. Pitarch G, Garde J, Torrijos A, et al: Adverse cutaneous reactions to erlotinib. Actas Dermosifiliogr 99:54-60, 2008

25. Lacouture ME, Wu S, Robert $\mathrm{C}$, et al: Evolving strategies for the management of hand-foot skin reaction associated with the multitargeted kinase inhibitors sorafenib and sunitinib. Oncologist 13: 1001-1011, 2008

26. Cheng $A L$, Kang $Y K$, Lin DY, et al: Sunitinib versus sorafenib in advanced hepatocellular carcinoma: Results of a randomized phase III trial. J Clin Oncol 31:4067-4075, 2013

27. Cainap C, Oin S, Huang WT, et al: Phase III trial of linifanib versus sorafenib in patients with advanced hepatocellular carcinoma (HCC). J Clin Oncol 31, 2013 (suppl 4; abstr 249)

28. Johnson PJ, Qin S, Park JW, et al: Brivanib versus sorafenib as first-line therapy in patients with unresectable, advanced hepatocellular carcinoma: Results from the randomized phase III BRISK-FL study. J Clin Oncol 31:3517-3524, 2013

29. Llovet JM, Decaens T, Raoul JL, et al: Brivanib in patients with advanced hepatocellular carcinoma who were intolerant to sorafenib or for whom sorafenib failed: Results from the randomized phase III BRISK-PS study. J Clin Oncol 31: 3509-3516, 2013

30. Llovet JM, Di Bisceglie AM, Bruix J, et al: Design and endpoints of clinical trials in hepatocellular carcinoma. J Natl Cancer Inst 100:698-711, 2008 